\title{
COVID-19
}

\section{Health Evidence Summary No.108}

\section{Kerry Millington \& Samantha Reddin}

Liverpool School of Tropical Medicine (LSTM) \& Institute of Development Studies 18 January 2021

This weekly COVID-19 health evidence summary (HES) is based on 3.5 hours of desk-based research. The summary is not intended to be a comprehensive summary of available evidence on COVID-19 but aims to make original documents easily accessible to decision makers which, if relevant to them, they should go to before making decisions.

\section{Clinical characteristics and management}

\begin{tabular}{|c|c|c|c|c|}
\hline $\begin{array}{l}\text { Publication } \\
\text { date }\end{array}$ & Title/URL & $\begin{array}{l}\text { Journal/Article } \\
\text { type }\end{array}$ & Summary & Keywords \\
\hline 11.01 .2021 & $\begin{array}{l}\text { Development } \\
\text { and } \\
\text { validation of } \\
\text { the ISARIC } \\
\text { 4C } \\
\text { deterioration } \\
\text { model for } \\
\text { adults } \\
\text { hospitalised } \\
\text { with COVID- } \\
\text { 19: a } \\
\text { prospective } \\
\text { cohort study }\end{array}$ & $\begin{array}{l}\text { The Lancet } \\
\text { Respiratory } \\
\text { Medicine | } \\
\text { Article }\end{array}$ & $\begin{array}{l}\text { Prognostic models to } \\
\text { predict the risk of clinical } \\
\text { deterioration in acute } \\
\text { COVID-19 cases are } \\
\text { urgently required to } \\
\text { inform clinical } \\
\text { management decisions } \\
\text { - Here a 4C Deterioration } \\
\text { model was developed } \\
\text { and validated, including } \\
11 \text { routinely measured } \\
\text { demographic, clinical, } \\
\text { and laboratory predictors, } \\
\text { for prediction of in- } \\
\text { hospital clinical } \\
\text { deterioration among } \\
74,944 \text { consecutive } \\
\text { adults recruited to the } \\
\text { ISARIC4C study across } \\
260 \text { hospitals in England, } \\
\text { Scotland and Wales. } \\
\text { The 4C Deterioration } \\
\text { model has strong } \\
\text { potential for clinical utility } \\
\text { and generalisability to } \\
\text { predict clinical } \\
\text { deterioration and inform } \\
\text { decision making among }\end{array}$ & $\begin{array}{l}\text { Prognostic } \\
\text { model, } \\
\text { clinical } \\
\text { deterioration }\end{array}$ \\
\hline
\end{tabular}




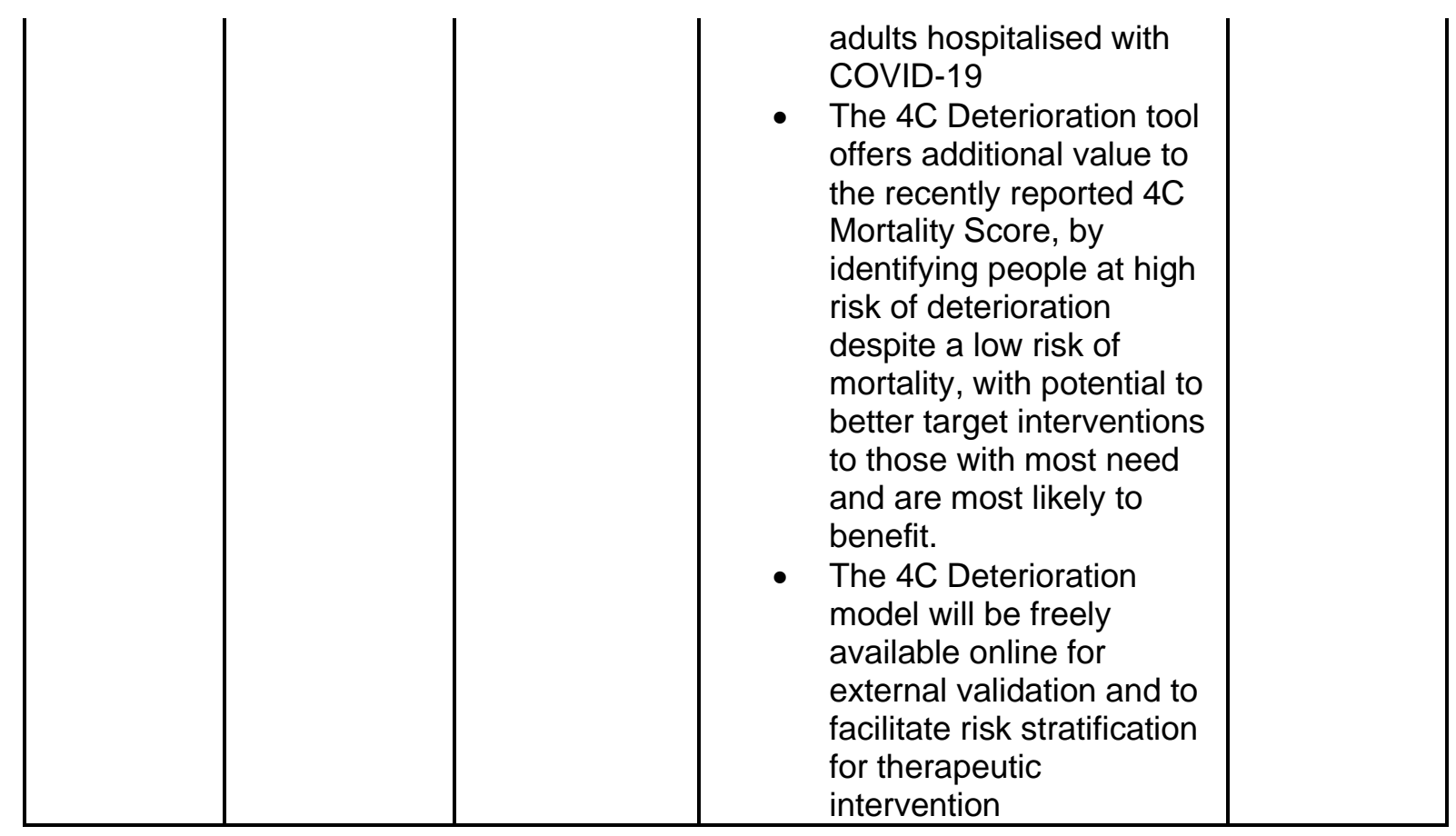

\section{Epidemiology and modelling}

\begin{tabular}{|c|c|c|c|c|}
\hline $\begin{array}{l}\text { Publication } \\
\text { date }\end{array}$ & Title/URL & $\begin{array}{l}\text { Journal/Article } \\
\text { type }\end{array}$ & Summary & Keywords \\
\hline 15.01 .2021 & $\begin{array}{l}\text { Characterisation } \\
\text { of the first } 250 \\
000 \text { hospital } \\
\text { admissions for } \\
\text { COVID-19 in } \\
\text { Brazil: a } \\
\text { retrospective } \\
\text { analysis of } \\
\text { nationwide data }\end{array}$ & $\begin{array}{l}\text { The Lancet } \\
\text { Respiratory } \\
\text { Medicine | } \\
\text { Article }\end{array}$ & $\begin{array}{l}\text { - Most LMICs have } \\
\text { little or no data } \\
\text { integrated into a } \\
\text { national } \\
\text { surveillance } \\
\text { system to identify } \\
\text { characteristics or } \\
\text { outcome of } \\
\text { COVID-19 } \\
\text { hospital } \\
\text { admission and the } \\
\text { impact of the } \\
\text { COVID-19 } \\
\text { pandemic on their } \\
\text { national health } \\
\text { systems } \\
\text { This retrospective } \\
\text { analysis of all } \\
\text { hospitalised } \\
\text { patients aged } 20 \\
\text { years or older } \\
\text { with RT-PCR- } \\
\text { confirmed } \\
\text { COVID-19 and } \\
\text { registered in a } \\
\text { nationwide } \\
\text { surveillance }\end{array}$ & $\begin{array}{l}\text { Clinical } \\
\text { outcome, } \\
\text { national } \\
\text { health system }\end{array}$ \\
\hline
\end{tabular}




\begin{tabular}{|c|c|c|c|c|c|}
\hline & & & & $\begin{array}{l}\text { database in Brazil } \\
\text { (SIVEP-Gripe). } \\
\text { Primary outcome: } \\
\text { in-hospital } \\
\text { mortality } \\
\text { Widespread } \\
\text { distribution of } \\
\text { COVID-19 across } \\
\text { all regions in } \\
\text { Brazil was } \\
\text { observed, } \\
\text { resulting in a high } \\
\text { overall disease } \\
\text { burden } \\
\text { In-hospital } \\
\text { mortality was } \\
\text { high, even in } \\
\text { patients younger } \\
\text { than } 60 \text { years, } \\
\text { and worsened by } \\
\text { existing regional } \\
\text { disparities within } \\
\text { the health system. } \\
\text { COVID-19 } \\
\text { disproportionately } \\
\text { affects not only } \\
\text { the most } \\
\text { vulnerable } \\
\text { patients but also } \\
\text { the most fragile } \\
\text { health systems. } \\
\text { Improvement in } \\
\text { access to high- } \\
\text { quality care for } \\
\text { critically ill } \\
\text { patients admitted } \\
\text { to hospital with } \\
\text { COVID-19 is } \\
\text { needed, } \\
\text { particularly in } \\
\text { LMICs } \\
\end{array}$ & \\
\hline 12.01.2021 & $\begin{array}{l}\text { First detection } \\
\text { of SARS-CoV-2 } \\
\text { spike protein } \\
\text { N501 mutation } \\
\text { in Italy in } \\
\text { August, } 2020\end{array}$ & $\begin{array}{l}\text { The Lancet } \\
\text { Infectious } \\
\text { Diseases | } \\
\text { Correspondence }\end{array}$ & & $\begin{array}{l}\text { A predecessor to } \\
\text { the B.1.1.7 "UK" } \\
\text { variant was seen } \\
\text { in Italy in August } \\
\text { (N501T } \\
\text { substitution) in a } \\
\text { 59-year-old man } \\
\text { with persistent } \\
\text { SARS-CoV-2 } \\
\text { infection } \\
\text { SARS-CoV-2 } \\
\text { strains with a } \\
\text { N501T } \\
\text { substitution might }\end{array}$ & UK variant \\
\hline
\end{tabular}




\begin{tabular}{|c|c|c|c|c|c|}
\hline & & & & $\begin{array}{l}\text { have circulated } \\
\text { unnoticed before } \\
\text { the end of Sept } \\
2020 \text {, when the } \\
\text { rapidly emerging } \\
\text { B.1.1.7 lineage } \\
\text { was first reported }\end{array}$ & \\
\hline 11.01 .2021 & $\begin{array}{l}\text { Estimates of } \\
\text { severity and } \\
\text { transmissibility } \\
\text { of novel South } \\
\text { Africa SARS- } \\
\text { CoV-2 variant } \\
501 Y . V 2\end{array}$ & $\begin{array}{l}\text { CMMID Github } \\
\text { repository | pre- } \\
\text { print (not yet } \\
\text { peer-reviewed) }\end{array}$ & & $\begin{array}{l}\text { On } 18 \text { Dec } 2020, \\
\text { researchers } \\
\text { reported } \\
\text { emergence of a } \\
\text { novel SARS-CoV- } \\
2 \text { variant, } \\
501 Y . V 2 \text {, in South } \\
\text { Africa } \\
\text { Using globally } \\
\text { available data, an } \\
\text { evaluation of the } \\
\text { novel variant for } \\
\text { potentially } \\
\text { increased } \\
\text { transmissibility or } \\
\text { immune escape is } \\
\text { reported here } \\
\text { Authors estimate } \\
501 Y . V 2 \text { is } 1.50 \\
\text { (95\% Crl } 1.20- \\
2.13) \text { times as } \\
\text { transmissible as } \\
\text { previously } \\
\text { circulating } \\
\text { variants } \\
\text { Some evidence of } \\
\text { a change in } \\
\text { severity was } \\
\text { found, though } \\
\text { there is } \\
\text { substantial } \\
\text { uncertainty } \\
\text { Along with the } \\
\text { emergence of a } \\
\text { novel UK variant } \\
\text { with similar } \\
\text { estimated } \\
\text { increased } \\
\text { transmissibility, } \\
\text { authors highlight } \\
\text { the substantial } \\
\text { challenges with } \\
\text { global control in } \\
\text { early } 2021, \text { the } \\
\text { need for } \\
\text { maintaining } \\
\text { control measures } \\
\text { and accelerating }\end{array}$ & $\begin{array}{l}\text { South African } \\
\text { variant, } \\
\text { disease } \\
\text { severity, } \\
\text { transmissibility }\end{array}$ \\
\hline
\end{tabular}




\begin{tabular}{|l|l|}
$\mid$ & vaccination roll- \\
out, and the \\
continued \\
monitoring of \\
vaccine \\
effectiveness \\
against novel \\
variants to detest \\
immune escape \\
promptly \\
\hline
\end{tabular}

\section{Testing}

\begin{tabular}{|c|c|c|c|c|}
\hline $\begin{array}{l}\text { Publication } \\
\text { date }\end{array}$ & Title/URL & $\begin{array}{l}\text { Journal/Article } \\
\text { type }\end{array}$ & Summary & Keywords \\
\hline 15.01 .2021 & $\begin{array}{l}\text { Comparison of } \\
\text { saliva and } \\
\text { nasopharyngeal } \\
\text { swab nucleic } \\
\text { acid } \\
\text { amplification } \\
\text { testing for } \\
\text { detection of } \\
\text { SARS-CoV-2 }\end{array}$ & $\begin{array}{l}\text { JAMA Internal } \\
\text { Medicine | } \\
\text { Systematic } \\
\text { review and } \\
\text { meta-analysis }\end{array}$ & $\begin{array}{l}\text { This systematic } \\
\text { review and meta- } \\
\text { analysis found that } \\
\text { saliva NAAT and } \\
\text { nasopharyngeal } \\
\text { NAAT had a similar } \\
\text { sensitivity and } \\
\text { specificity } \\
\text { Given the ease of } \\
\text { use and comparable } \\
\text { diagnostic accuracy, } \\
\text { saliva NAAT offers } \\
\text { an attractive } \\
\text { alterative to } \\
\text { nasopharyngeal } \\
\text { swab NAAT which } \\
\text { may help increase } \\
\text { mass testing efforts }\end{array}$ & $\begin{array}{l}\text { Saliva testing, } \\
\text { nasopharyngeal } \\
\text { testing }\end{array}$ \\
\hline
\end{tabular}

\section{Therapeutics}

\begin{tabular}{|c|c|c|c|c|}
\hline $\begin{array}{l}\text { Publication } \\
\text { date }\end{array}$ & Title/URL & $\begin{array}{l}\text { Journal/Article } \\
\text { type }\end{array}$ & Summary & Keywords \\
\hline 15.01 .2021 & $\begin{array}{l}\text { RECOVERY } \\
\text { trial closes } \\
\text { recruitment to } \\
\text { convalescent } \\
\text { plasma } \\
\text { treatment for } \\
\text { patients } \\
\text { hospitalised }\end{array}$ & $\begin{array}{l}\text { Statement } \\
\text { from the } \\
\text { RECOVERY } \\
\text { trial chief } \\
\text { investigators }\end{array}$ & $\begin{array}{l}\text { The DMC of the } \\
\text { RECOVERY trial } \\
\text { reviewed data on } \\
\text { patients randomised to } \\
\text { convalescent plasma vs } \\
\text { usual care. Preliminary } \\
\text { analysis of } 10,406 \\
\text { randomised patients } \\
\text { with } 1873 \text { reported } \\
\text { deaths shows no } \\
\text { significant difference in }\end{array}$ & $\begin{array}{l}\text { Convalescent } \\
\text { plasma }\end{array}$ \\
\hline
\end{tabular}




\begin{tabular}{|c|c|c|c|c|}
\hline & $\begin{array}{l}\text { with COVID- } \\
19\end{array}$ & & $\begin{array}{l}\text { the primary endpoint of } \\
28 \text {-day mortality } \\
\text { - } 18 \% \text { convalescent } \\
\text { plasma vs } 18 \% \text { usual } \\
\text { care alone; RR } 1.04 \\
{[95 \% \mathrm{Cl} 0.95-1.14] ;} \\
\mathrm{p}=0.34 \\
\text { Follow-up of patients is } \\
\text { ongoing and final } \\
\text { results will be published } \\
\text { as soon as possible } \\
\text { Whilst the overall result } \\
\text { is negative, full results } \\
\text { are required to } \\
\text { determine if there is any } \\
\text { benefit in particular } \\
\text { patient subgroups } \\
\text { Note RECOVERY } \\
\text { continues to study the } \\
\text { REGN-CoV2 } \\
\text { monoclonal antibody } \\
\text { cocktail - shown to } \\
\text { have benefit in RCTs in } \\
\text { early COVID. In Ebola, } \\
\text { convalescent plasma } \\
\text { did not work but } \\
\text { monoclonal antibodies } \\
\text { did. }\end{array}$ & \\
\hline 13.01 .2021 & $\begin{array}{l}\text { Convalescent } \\
\text { plasma } \\
\text { antibody } \\
\text { levels and the } \\
\text { risk of deaths } \\
\text { from Covid-19 }\end{array}$ & NEJM | Article & $\begin{array}{l}\text { A retrospective study } \\
\text { based on a U.S. } \\
\text { national registry, of anti- } \\
\text { SARS-CoV-2 IgG } \\
\text { antibody levels in } \\
\text { convalescent plasma } \\
\text { used to treat } 3082 \\
\text { hospitalised adults with } \\
\text { Covid-19. Primary } \\
\text { outcome was death } \\
\text { within } 30 \text { days after } \\
\text { plasma transfusion } \\
\text { No effect on the risk of } \\
\text { death was observed } \\
\text { among patients who } \\
\text { had received } \\
\text { mechanical ventilation } \\
\text { Among patients who } \\
\text { were not receiving } \\
\text { mechanical ventilation, } \\
\text { transfusion of plasma } \\
\text { with higher anti-SARS- } \\
\text { CoV-2 IgG antibody } \\
\text { levels was associated } \\
\text { with a lower risk of } \\
\text { death than transfusion }\end{array}$ & $\begin{array}{l}\text { Convalescent } \\
\text { plasma }\end{array}$ \\
\hline
\end{tabular}




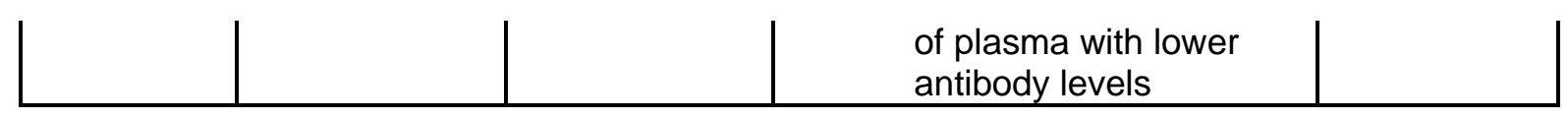

\section{Vaccines}

\begin{tabular}{|c|c|c|c|c|}
\hline $\begin{array}{l}\text { Publication } \\
\text { date }\end{array}$ & Title/URL & $\begin{array}{l}\text { Journal/Article } \\
\text { type }\end{array}$ & Summary & Keywords \\
\hline 13.01 .2021 & $\begin{array}{l}\text { Interim results } \\
\text { of a phase 1- } \\
2 \text { a trial of } \\
\text { Ad26.COV2.S } \\
\text { Covid-19 } \\
\text { vaccine }\end{array}$ & NEJM | Article & $\begin{array}{l}\text { Candidate vaccine, } \\
\text { Ad26.COV2.S, is a } \\
\text { recombinant, } \\
\text { replication- } \\
\text { incompetent } \\
\text { adenovirus serotype } \\
26 \text { vector encoding a } \\
\text { full-length and } \\
\text { stabilised SARS-CoV- } \\
2 \text { spike protein } \\
\text { Multicentre, placebo- } \\
\text { controlled, phase 1-2a } \\
\text { trial of two age groups } \\
\text { receiving a low or high } \\
\text { dose of the vaccine } \\
\text { candidate or placebo } \\
\text { in a single-dose or } \\
\text { two-dose schedule } \\
\text { The safety and } \\
\text { immunogenicity } \\
\text { profiles support further } \\
\text { development of this } \\
\text { vaccine candidate }\end{array}$ & $\begin{array}{l}\text { Vaccine } \\
\text { candidate, } \\
\text { Ad26.COV2.S }\end{array}$ \\
\hline
\end{tabular}




\section{Comments, Editorials, Opinions, Blogs, News}

\begin{tabular}{|c|c|c|}
\hline $\begin{array}{l}\text { Publication } \\
\text { date }\end{array}$ & Title/URL & Journal | Article type \\
\hline 18.01 .2021 & $\begin{array}{l}\text { UK funded research essential to get vaccines to } \\
\text { the poorest in the world }\end{array}$ & $\begin{array}{l}\text { Open Access Government I } \\
\text { Blog }\end{array}$ \\
\hline 17.01 .2021 & $\begin{array}{l}\text { Equitable recovery from COVID-19: bring global } \\
\text { commitments to community level }\end{array}$ & $\begin{array}{l}\text { BMJ Global Health । } \\
\text { Commentary }\end{array}$ \\
\hline 15.01 .2021 & $\begin{array}{l}\text { China COVID vaccine reports mixed results - } \\
\text { what does that mean for the pandemic? }\end{array}$ & Nature | News \\
\hline 13.01.2021 & 2021: a new year for the WHO & Nature Medicine | Editorial \\
\hline 13.01.2021 & $\begin{array}{l}\text { Women and children last? Shaking up exclusion } \\
\text { criteria for vaccine trials }\end{array}$ & Nature Medicine | World View \\
\hline 13.01 .2021 & On the origins of SARS-CoV-2 & Nature Medicine | World View \\
\hline 13.01.2021 & $\begin{array}{l}\text { Immune determinants of COVID-19 disease } \\
\text { presentation and severity }\end{array}$ & Nature Medicine | Perspective \\
\hline
\end{tabular}




\section{Dashboards \& Trackers}

\begin{tabular}{|c|c|c|c|c|c|c|c|}
\hline $\begin{array}{l}\text { Cases \& deaths: } \\
\text { Global }\end{array}$ & $\begin{array}{l}\text { Cases } \quad \& \\
\text { deaths: } \\
\text { Regional }\end{array}$ & $\begin{array}{l}\text { Cases } \quad \& \\
\text { deaths: } \\
\text { Country }\end{array}$ & $\begin{array}{l}\text { Living evidence \& } \\
\text { policy maps }\end{array}$ & $\begin{array}{l}\text { Current research } \\
\text { including trials }\end{array}$ & Diagnostics & Treatments & Vaccines \\
\hline WHO sitreps & WHO Africa & Ghana & COVID-NMA & WHO & $\begin{array}{l}\text { FIND SARS- } \\
\text { CoV-2 Test } \\
\text { Tracker }\end{array}$ & $\begin{array}{l}\text { Global COVID- } \\
19 \text { Clinical Trial } \\
\text { Tracker }\end{array}$ & CEPI \\
\hline WHO dashboard & $\begin{array}{l}\text { African } \\
\text { Arguments }\end{array}$ & Indonesia & EPPI Centre & $\begin{array}{l}\text { WHO International } \\
\text { Clinical Trials } \\
\text { Registry Platform } \\
\text { (ICTRP) }\end{array}$ & $\begin{array}{l}\text { FIND SARS- } \\
\text { CoV-2 } \\
\text { Diagnostics: } \\
\text { performance } \\
\text { data }\end{array}$ & $\begin{array}{l}\text { US NIH } \\
\text { registered } \\
\text { clinical trials }\end{array}$ & $\begin{array}{l}\text { Vaccine } \\
\text { Centre LSHTM }\end{array}$ \\
\hline $\begin{array}{l}\text { Johns Hopkins } \\
\text { University }\end{array}$ & $\begin{array}{l}\text { European } \\
\text { CDC }\end{array}$ & Nigeria CDC & $\begin{array}{l}\text { Norwegian } \\
\text { Institute of Public } \\
\text { Health }\end{array}$ & Cytel & $\begin{array}{l}\text { Serology-based } \\
\text { tests for COVID- } \\
19\end{array}$ & Solidarity trial & $\begin{array}{l}\text { COVID-19 } \\
\text { Oxford } \\
\text { Vaccine Trial }\end{array}$ \\
\hline WEF & & Sierra Leone & $\begin{array}{l}\text { Oxford C19 } \\
\text { Government } \\
\text { Response } \\
\text { Tracker } \\
\text { (OxCGRT) }\end{array}$ & US NIH & $\begin{array}{l}\text { Our World in } \\
\text { Data: C19 } \\
\text { Testing }\end{array}$ & $\begin{array}{l}\text { COVID-19 } \\
\text { Therapeutics } \\
\text { Accelerator }\end{array}$ & $\begin{array}{l}\text { COVID-19 } \\
\text { Vaccine } \\
\text { Tracker }\end{array}$ \\
\hline
\end{tabular}




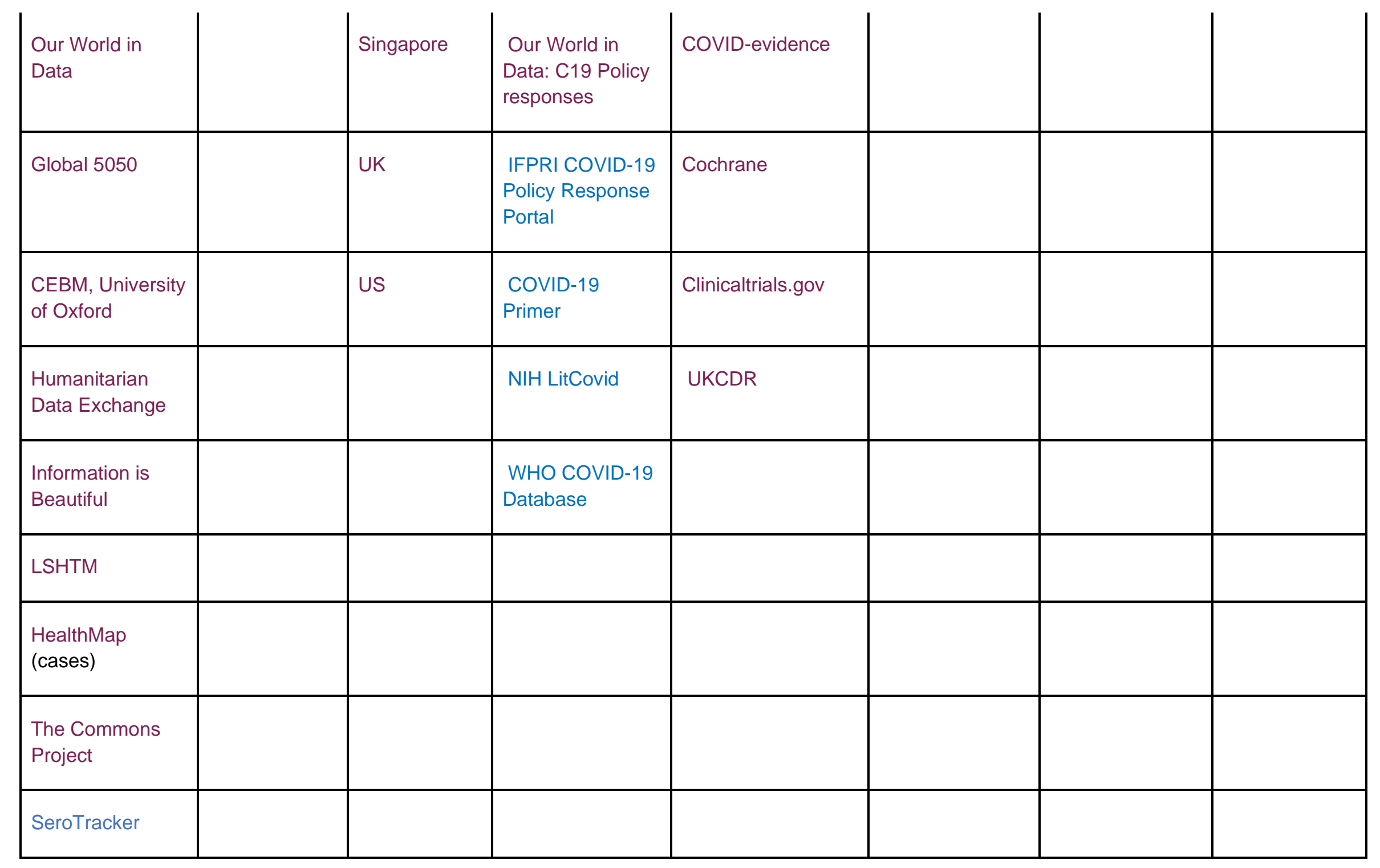




\section{C19 Resource Hubs}

\begin{tabular}{|c|c|c|c|c|c|}
\hline Global & $\begin{array}{l}\text { Regional } \\
\text { \& Country }\end{array}$ & $\begin{array}{l}\text { Academi } \\
\text { C } \\
\text { journals } \\
\text { \& } \\
\text { Publisher } \\
\text { s }\end{array}$ & $\begin{array}{l}\text { Institutes/Centres/Funders/ } \\
\text { Other }\end{array}$ & $\begin{array}{l}\text { Health } \\
\text { Topics }\end{array}$ & $\begin{array}{l}\text { Social } \\
\text { Sciences }\end{array}$ \\
\hline $\begin{array}{l}\text { WHO } \\
\text { COVID-19 } \\
\text { pandemic }\end{array}$ & $\begin{array}{l}\text { Africa } \\
\text { CDC }\end{array}$ & $\begin{array}{l}\text { Annals of } \\
\text { Internal } \\
\text { Medicine }\end{array}$ & LSTM & $\begin{array}{l}\text { Stop TB } \\
\text { Partnership }\end{array}$ & SSHAP \\
\hline $\begin{array}{l}\text { WHO risk } \\
\text { communicati } \\
\text { on }\end{array}$ & $\begin{array}{l}\text { African } \\
\text { Union }\end{array}$ & BMJ & LSHTM & & IDA \\
\hline WHO Q\&A & $\begin{array}{l}\text { Nigeria } \\
\text { CDC }\end{array}$ & $\begin{array}{l}\text { Bulletin } \\
\text { of the } \\
\text { WHO }\end{array}$ & $\begin{array}{l}\text { ICL MRC Centre for Global } \\
\text { Infectious Disease Analysis }\end{array}$ & $\begin{array}{l}\text { Global } \\
\text { Menstrual } \\
\text { Collective }\end{array}$ & $\begin{array}{l}\text { Disability } \\
\text { and } \\
\text { inclusion }\end{array}$ \\
\hline $\begin{array}{l}\text { WHO Global } \\
\text { research }\end{array}$ & $\begin{array}{l}\text { GeoPoll: } \\
\text { SSA }\end{array}$ & $\begin{array}{l}\text { Cambrid } \\
\text { ge } \\
\text { Universit } \\
\text { y Press }\end{array}$ & ODI & $\begin{array}{l}\text { SLH: } \\
\text { Handwashi } \\
\text { ng in low } \\
\text { resource } \\
\text { settings }\end{array}$ & $\begin{array}{l}\text { Coregrou } \\
\text { p IDDC }\end{array}$ \\
\hline $\begin{array}{l}\text { COVID-19 } \\
\text { Solidarity } \\
\text { Response } \\
\text { Fund }\end{array}$ & $\begin{array}{l}\text { Global } \\
\text { Health } \\
\text { Network } \\
\text { Africa }\end{array}$ & $\begin{array}{l}\text { Cell } \\
\text { Press }\end{array}$ & Johns Hopkins University & $\begin{array}{l}\text { RBM } \\
\text { Partnership }\end{array}$ & $\begin{array}{l}\text { Ethics, } \\
\text { health } \\
\text { systems \& } \\
\text { COVID-19 }\end{array}$ \\
\hline UN & $\begin{array}{l}\text { African } \\
\text { Academy } \\
\text { of } \\
\text { Sciences }\end{array}$ & $\begin{array}{l}\text { Cochran } \\
\text { e }\end{array}$ & $\begin{array}{l}\text { Center for Global } \\
\text { Development }\end{array}$ & $\begin{array}{l}\text { Epidemic } \\
\text { Preparedne } \\
\text { ss } \\
\text { Innovations }\end{array}$ & $\begin{array}{l}\text { Social } \\
\text { Developm } \\
\text { ent Direct } \\
\text { C19 blog } \\
\text { series }\end{array}$ \\
\hline UN Women & $\begin{array}{l}\text { Africa } \\
\text { Evidence } \\
\text { Network }\end{array}$ & Elsevier & CMMID Repository & & \\
\hline
\end{tabular}




\begin{tabular}{|c|c|c|c|c|}
\hline UNOCHA & $\begin{array}{l}\text { OCHA } \\
\text { Southern } \\
\text { and } \\
\text { Eastern } \\
\text { Africa } \\
\text { COVID-19 } \\
\text { Digest }\end{array}$ & $\begin{array}{l}\text { Health } \\
\text { Policy } \\
\text { and } \\
\text { Planning }\end{array}$ & $\begin{array}{l}\text { Norwegian Institute of } \\
\text { Public Health }\end{array}$ & \\
\hline UNHCR & $\begin{array}{l}\text { South } \\
\text { African } \\
\text { Governme } \\
\text { nt }\end{array}$ & $\begin{array}{l}\text { JAMA } \\
\text { Network }\end{array}$ & $\begin{array}{l}\text { Oxford Centre for } \\
\text { Evidence-based Medicine }\end{array}$ & \\
\hline UNICEF & & $\begin{array}{l}\text { The } \\
\text { Lancet }\end{array}$ & HEART & \\
\hline UNESCO & & $\begin{array}{l}\text { medRxiv } \\
\text { and } \\
\text { bioRxiv } \\
\text { (Preprint } \\
\text { s) }\end{array}$ & UKRI & \\
\hline UN WFP & & NEJM & Evidence Aid & \\
\hline GOARN & & $\begin{array}{l}\text { Oxford } \\
\text { Universit } \\
\text { y Press }\end{array}$ & $\mathrm{NIH}$ & \\
\hline EPI-WIN & & PLoS & $\begin{array}{l}\text { IFPRI Resources and } \\
\text { Analyses of C19 Impact }\end{array}$ & \\
\hline World Bank & & $\begin{array}{l}\text { SAGE } \\
\text { journals }\end{array}$ & Prevent Epidemics & \\
\hline $\begin{array}{l}\text { Our World in } \\
\text { Data }\end{array}$ & & Science & & \\
\hline $\begin{array}{l}\text { COVID-19 } \\
\text { Narratives by } \\
\text { David } \\
\text { Nabarro }\end{array}$ & & $\begin{array}{l}\text { Springer } \\
\text { Nature }\end{array}$ & & \\
\hline
\end{tabular}




\begin{tabular}{|c|c|c|c|}
\hline Reliefweb & $\begin{array}{l}\text { SSRN } \\
\text { (Preprint } \\
\text { s) }\end{array}$ & & \\
\hline $\begin{array}{l}\text { Humanitarian } \\
\text { OpenStreetM } \\
\text { ap Team }\end{array}$ & Wiley & & \\
\hline $\begin{array}{l}\text { Global } \\
\text { Partnership } \\
\text { for } \\
\text { Sustainable } \\
\text { Development } \\
\text { Data }\end{array}$ & & & \\
\hline WorldPop & & 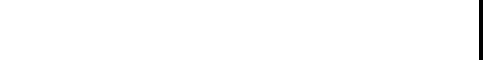 & \\
\hline Flowminder & & & \\
\hline COVID-END & & & \\
\hline $\begin{array}{l}\text { Premise } \\
\text { COVID-19 } \\
\text { Global } \\
\text { Impact Study }\end{array}$ & & & \\
\hline GISAID & & & \\
\hline
\end{tabular}




\section{Online learning \& events}

\begin{tabular}{|c|c|c|c|c|}
\hline Date & Title/URL & $\begin{array}{l}\text { Online } \\
\text { learning/event }\end{array}$ & Duration & Lead \\
\hline 14.01 .2021 & $\begin{array}{l}\text { Evidence to impact in } \\
\text { crisis: how have we } \\
\text { measured up during } \\
\text { the COVID-19 } \\
\text { pandemic? }\end{array}$ & Webinar & $1 \mathrm{~h} 30$ & CGD \\
\hline 04.12 .2020 & $\begin{array}{l}\text { COVID-19, supply } \\
\text { chain resilience and } \\
\text { global trade }\end{array}$ & Webinar & $1 \mathrm{~h}$ & CGD \\
\hline 03.12 .2020 & $\begin{array}{l}\text { More money for } \\
\text { health services: What } \\
\text { is the tole of PFM in } \\
\text { the "new normal"? }\end{array}$ & $\begin{array}{l}\text { WHO \& CGD } \\
\text { Health systems } \\
\text { Governance \& } \\
\text { Financing }\end{array}$ & $1 \mathrm{~h} 30$ & Joe Kutzin \\
\hline 01.12 .2020 & $\begin{array}{l}\text { Solutions and support } \\
\text { for the mental } \\
\text { wellbeing of } \\
\text { community health } \\
\text { workers on the } \\
\text { COVID-19 frontline }\end{array}$ & Webinar & & $\begin{array}{l}\text { HSG TWG on CHWs } \\
\text { with The George } \\
\text { Institute for Global } \\
\text { Health }\end{array}$ \\
\hline 19.11 .2020 & $\begin{array}{l}\text { Looking at the } \\
\text { pandemic with a } \\
\text { gender lens }\end{array}$ & $\begin{array}{l}\text { Live Twitter } \\
\text { conversation }\end{array}$ & & SSHAP \\
\hline 16.11 .2020 & $\begin{array}{l}\text { HIFA and WHO } \\
\text { collaborate to } \\
\text { promote sharing of } \\
\text { experience and } \\
\text { expertise around the } \\
\text { maintenance of } \\
\text { essential health } \\
\text { services during (and } \\
\text { after) the pandemic }\end{array}$ & $\begin{array}{l}\text { 4-week } \\
\text { discussion } \\
\text { starting } 16 \text { Nov }\end{array}$ & & HIFA \\
\hline 10.11 .2020 & $\begin{array}{l}\text { COVID-19 vaccine } \\
\text { predictions part } 2 \text { : } \\
\text { estimating the time }\end{array}$ & Online event & $1 \mathrm{~h} 30$ & CGD \\
\hline
\end{tabular}




\begin{tabular}{|c|c|c|c|c|}
\hline & $\begin{array}{l}\text { before we approve } \\
\text { efficacious COVID-19 } \\
\text { vaccines }\end{array}$ & & & \\
\hline 16.10 .2020 & $\begin{array}{l}\text { Financing a Global } \\
\text { Public Health } \\
\text { Response }\end{array}$ & Online event & $1 \mathrm{~h} 30$ & CGD \\
\hline 02.10 .2020 & $\begin{array}{l}\text { Understanding and } \\
\text { Improving COVID-19 } \\
\text { Vaccine Portfolio }\end{array}$ & Online event & 1h30 & CGD \\
\hline 21.09 .2020 & $\begin{array}{l}\text { Mitigating the } \\
\text { Economic and Health } \\
\text { Impact of COVID-19 } \\
\text { across Africa }\end{array}$ & Online event & $1 \mathrm{~h} 30$ & CGD, GF, AU \\
\hline June 2020 & $\begin{array}{l}\text { OpenWHO, the free, } \\
\text { open-access learning } \\
\text { platform for health } \\
\text { emergencies, now } \\
\text { offers } 10 \text { online } \\
\text { courses related to } \\
\text { COVID19. }\end{array}$ & Online courses & Varies & $\mathrm{WHO}$ \\
\hline $\begin{array}{l}\text { Available } \\
\text { now }\end{array}$ & $\begin{array}{l}\text { Standard } \\
\text { precautions: } \\
\text { Environmental } \\
\text { cleaning and } \\
\text { disinfection }\end{array}$ & Online course & 1 hour & WHO \\
\hline $\begin{array}{l}\text { Available } \\
\text { now }\end{array}$ & $\begin{array}{l}\text { COVID-19: Effective } \\
\text { Nursing in Times of } \\
\text { Crisis }\end{array}$ & Online course & $\begin{array}{l}2 \text { weeks - } \\
2 \text { hours } \\
\text { per week }\end{array}$ & $\begin{array}{l}\text { Johns Hopkins School } \\
\text { of Nursing }\end{array}$ \\
\hline $\begin{array}{l}\text { Available } \\
\text { now }\end{array}$ & $\begin{array}{l}\text { WHO Academy and } \\
\text { WHO Info mobile } \\
\text { applications }\end{array}$ & Mobile app & & WHO \\
\hline $\begin{array}{l}\text { Available } \\
\text { now }\end{array}$ & $\begin{array}{l}\text { COVID-19: } \\
\text { Pandemics, } \\
\text { Modelling and Policy }\end{array}$ & Online learning & $\begin{array}{l}2 \text { weeks | } \\
2 \text { hours } \\
\text { weekly } \\
\text { study }\end{array}$ & $\begin{array}{l}\text { FutureLearn UNESCO } \\
\text { UNITWIN Complex } \\
\text { Systems Digital } \\
\text { Campus/Open } \\
\text { University }\end{array}$ \\
\hline
\end{tabular}




\begin{tabular}{|c|c|c|c|c|}
\hline 11.5 .2020 & $\begin{array}{l}\text { COVID-19 Contact } \\
\text { Tracing course }\end{array}$ & Online learning & 5 hours & $\begin{array}{l}\text { Johns Hopkins } \\
\text { Bloomberg School of } \\
\text { Health }\end{array}$ \\
\hline $\begin{array}{l}7-28 \text { May } \\
2020\end{array}$ & $\begin{array}{l}\text { Virtual Evidence } \\
\text { Weeks }\end{array}$ & 5 sessions & $1 \mathrm{~h} 30$ & $\begin{array}{l}\text { International Initiative } \\
\text { for Impact Evaluation } \\
\text { (3ie) }\end{array}$ \\
\hline $\begin{array}{l}\text { Tuesdays } \\
\text { at } 1700 \\
\text { CEST } \\
\text { (Geneva } \\
\text { time) \& } \\
\text { Thursdays } \\
0830 \text { CEST } \\
\text { (Geneva } \\
\text { time) }\end{array}$ & $\begin{array}{l}\text { COVID-19 Open } \\
\text { online brief with Dr } \\
\text { David Nabarro }\end{array}$ & Event & $1 \mathrm{~h}$ & $4 S D$ \\
\hline $\begin{array}{l}\text { Available } \\
\text { now }\end{array}$ & $\begin{array}{l}\text { Emerging respiratory } \\
\text { viruses, including } \\
\text { COVID-19: methods } \\
\text { for detection, } \\
\text { prevention, response } \\
\text { and control }\end{array}$ & Online learning & 3 hours & WHO \\
\hline $\begin{array}{l}\text { Available } \\
\text { now }\end{array}$ & $\begin{array}{l}\text { Responding to } \\
\text { COVID-19: Real-time } \\
\text { training for the } \\
\text { coronavirus disease } \\
\text { outbreak }\end{array}$ & Online learning & $\begin{array}{l}\text { Multiple } \\
\text { self-paced } \\
\text { course }\end{array}$ & WHO \\
\hline $\begin{array}{l}25 \text { May } \\
2020\end{array}$ & $\begin{array}{l}\text { COVID-19: Tackling } \\
\text { the Novel } \\
\text { Coronavirus }\end{array}$ & Online learning & $\begin{array}{l}3 \text { weeks | } \\
4 \text { hours } \\
\text { weekly } \\
\text { study }\end{array}$ & $\begin{array}{l}\text { FutureLearn } \\
\text { LSHTM/UK PHRST }\end{array}$ \\
\hline $\begin{array}{l}\text { Available } \\
\text { online now } \\
\text { without } \\
\text { mentors. } \\
\text { Updated } \\
\text { version will } \\
\text { commence }\end{array}$ & $\begin{array}{l}\text { COVID-19 } \\
\text { Diagnostics and } \\
\text { Testing }\end{array}$ & Online learning & $\begin{array}{l}3 \text { weeks | } \\
3 \text { hours } \\
\text { weekly } \\
\text { study }\end{array}$ & $\begin{array}{l}\text { FutureLearn } \\
\text { FIND/LSHTM/ASLM }\end{array}$ \\
\hline
\end{tabular}




\begin{tabular}{|l|l|l|l|l|}
$\begin{array}{l}\text { early June } \\
2020\end{array}$ & & & \\
\hline 6 April 2020 & $\begin{array}{l}\text { COVID-19 Critical } \\
\text { Care: Understanding } \\
\text { and Application }\end{array}$ & Online learning & $\begin{array}{l}5 \text { weeks } \\
1 \text { hour } \\
\text { weekly } \\
\text { study }\end{array}$ & $\begin{array}{l}\text { FutureLearn University } \\
\text { of Edinburgh \& Royal } \\
\text { College of Physicians } \\
\text { of Edinburgh }\end{array}$ \\
\hline $\begin{array}{l}\text { Available } \\
\text { now }\end{array}$ & $\begin{array}{l}\text { COVID-19 supporting } \\
\text { online courses }\end{array}$ & Online learning & $\begin{array}{l}\text { Multiple } \\
\text { self-paced } \\
\text { course }\end{array}$ & BMJ Learning \\
\hline
\end{tabular}

\section{Suggested citation}

Millington, K.A. and Reddin, S. (2021). COVID-19 Health Evidence Summary No.108. K4D

Evidence Summary. Brighton, UK: Institute of Development Studies. DOI:

10.19088/K4D.2021.007

\section{Rapid review methodology}

The rapid weekly search for peer-reviewed literature is carried out through a PubMed search with the following keywords ("COVID-19" OR "severe acute respiratory syndrome coronavirus 2" OR "2019-nCoV" OR "SARS-CoV2" OR "2019nCoV" OR "coronavirus" ) AND ("Africa" OR "South Asia" OR "Developing" OR "low-income" OR "low income" OR "lower-middle income" OR "low and middle income" OR "LMIC" OR "LIC" OR "global south") OR ("poverty") OR ("equity" OR "equities"), restricted to articles published in the previous 2 to 3 days, in English. This is complemented by a search of the homepage of the following high-impact global health journals: The Lancet journals, New England Journal of Medicine, Nature, JAMA, Annals of Internal Medicine, Cochrane Reviews, BMJ Global Health, the PLoS journals and a Twitter search of their Twitter pages. A search also of preprints from bioRxiv and medRxiv. Please note that papers that have not been peer-reviewed are highlighted in red. All primary research papers that relate to the primary and secondary impacts of the COVID-19 response in LMICs, and disease control and health system responses are included. Articles related to tackling the secondary impacts on other sectors are not included. Additional commentaries, opinions, and commissioned pieces are selected based on relevance.

The search for dashboards, guidelines, tools, editorials, comments, blogs, opinions and news is through the academic journals listed above, C19 resource hubs and following lead academics and professionals on Twitter. 


\section{About this report}

This weekly COVID-19 health evidence summary (HES) is based on 3.5 hours of desk-based research. The summary is not intended to be a comprehensive summary of available evidence on COVID-19 but aims to make original documents easily accessible to decision makers which, if relevant to them, they should go to before making decisions. The HES are not intended to replace medical or professional advice and the researcher or the K4D consortium cannot be held responsible for any decisions made about COVID-19 on the basis of the HES alone. K4D services are provided by a consortium of leading organisations working in international development, led by the Institute of Development Studies (IDS), with Education Development Trust, Itad, University of Leeds Nuffield Centre for International Health and Development, Liverpool School of Tropical Medicine (LSTM), University of Birmingham International Development Department (IDD) and the University of Manchester Humanitarian and Conflict Response Institute (HCRI).

This evidence summary was prepared for the UK Government's Foreign, Commonwealth and Development Office (FCDO) and its partners in support of pro-poor programmes. Except where otherwise stated, it is licensed for non-commercial purposes under the terms of the Open Government Licence v3.0. K4D cannot be held responsible for errors, omissions or any consequences arising from the use of information contained in this health evidence summary. Any views and opinions expressed do not necessarily reflect those of FCDO, K4D or any other contributing organisation.

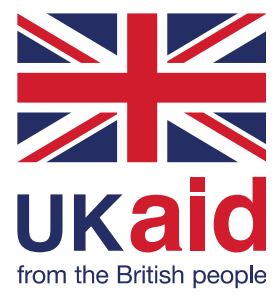

(c) Crown copyright 2021 